\title{
EFEITOS DO USO DO SOLO SOBRE RIACHOS DE CABECEIRA NA BACIA DO RIO CUIABÁ, MATO GROSSO
}

\author{
Effects of the Land Use over the Headwater \\ Streams on the Cuiabá River Basin, Mato Grosso
}

Nadja Gomes Machado ${ }^{1,3}$, Mariano Martínez Espinosa², Marcelo Sacardi Biudes ${ }^{3}$ e Jerry Magno Ferreira Penha ${ }^{4}$ nadja.machado@blv.ifmt.edu.br

' Laboratório de Biologia da Conservação (LABIC), Instituto Federal de Mato Grosso (IFMT), Cuiabá, MT, Brazil.

${ }^{2}$ Departamento de Estatística, Universidade Federal de Mato Grosso (UFMT), Cuiabá, MT, Brazil.

3 Programa de Pós-graduação em Física Ambiental, Universidade Federal de Mato Grosso (UFMT), Cuiabá, MT, Brazil.

${ }_{4}^{4}$ Departamento de Botânica e Ecologia, Universidade Federal de Mato Grosso (UFMT), Cuiabá, MT, Brazil.

\section{Resumo}

Os riachos de cabeceira são influenciados pelo ambiente terrestre, o que os tornam vulneráveis as atividades antrópicas, principalmente em áreas de cerrado com intensa modificação causada pelo uso do solo. O objetivo deste trabalho foi avaliar o efeito do uso do solo sobre atributos de riachos de cabeceira na bacia do Rio Cuiabá, Mato Grosso. Os 26 riachos amostrados foram caracterizados a partir de imagens de satélite e medidas in loco. Os atributos medidos foram tipo de uso de solo, oxigênio dissolvido, $\mathrm{pH}$, temperatura, turbidez, largura e profundidade do canal, velocidade e tipo de substrato. A turbidez afetou negativamente o oxigênio dissol- 
vido em riachos de cabeceira, assim como a largura do canal afetou a temperatura e a largura da mata ciliar afetou positivamente a quantidade de folhas. Portanto, o uso do solo afetou direta e indiretamente atributos físicos do canal e químicos da água de riachos de cabeceira de Cerrado. Palavras-chave: Cerrado; Hidrogeomorfologia; Impactos Ambientais. Abstract

The headwaters streams are influenced by the terrestrial environment, which makes them vulnerable to human activities, especially in cerrado areas with intense changes caused by land use. The aim of this study was to evaluate the effect of land use attributes on headwater streams in the Rio Cuiabá basin, Mato Grosso. The 26 sampled streams were characterized from satellite images and measurements in situ. The attributes measured were type of land use in the watershed, dissolved oxygen, $\mathrm{pH}$, temperature, turbidity, riparian width, average width, average depth, velocity and substrate type on the channel streams. The turbidity affected negatively dissolved oxygen in headwater streams, as well as the channel width positively affected water temperature and the riparian width positively affected leaves. Therefore, land use affected directly and indirectly attributes of headwater streams in Cerrado.

Keywords: Savanna; Hydrogeomorphology; Environmental impacts.

\section{Introdução}

Um riacho pode ser definido como um ecossistema biofísico que resulta, incorpora e depende do volume de água canalizado e da dinâmica de transporte de sedimentos associados, cuja gênese surge dos processos físicos de formação do canal, causado pela convergência do fluxo das águas superficiais e da emergência de água do solo para a superfície (Poole, 2010). As disciplinas de geomorfologia, hidrologia e hidrogeologia tiveram uma influência marcante sobre a evolução da concepção de riacho como um continuum, um corredor, uma rede ou uma paisagem (Poole, 2010).

Recentemente, rios e riachos são estudados tanto na perspectiva da paisagem propriamente dita como ecossistemas fortemente influenciados por áreas adjacentes em múltiplas escalas (Allan, 2004). A ecologia de paisagem enfatiza a heterogeneidade de habitats, a conectividade e a escala nos estudos sobre águas correntes. Se riachos possuem uma escala espacial hierárquica (Lowe et al., 2006), podemos explicitá-la da 
maior escala espacial da paisagem, denominada de bacia, até em escalas sucessivamente menores do canal como unidades individuais (corredeiras e piscinas), e micro-habitat (Fausch et al., 2002).

Vale ressaltar que existe forte relação entre o ambiente terrestre e aquático, uma vez que os riachos de cabeceira são influenciados pelo ambiente terrestre por causa de seu baixo quociente entre área do canal e sua área de drenagem (Karr \& Schlosser, 1978). Por exemplo, a retirada da vegetação nativa altera o ciclo hidrológico (Oliveira et al., 2005), a entrada de matéria e energia nos riachos (Allan 1995), os processos de erosão e deposição de seus canais (Church, 2002; Wiens 2002) e, consequentemente, as populações de peixes que lá ocorrem (Fausch et al., 2002).

As condições geomorfológicas de um riacho é o resultado combinado de muitos processos físicos individuais, os quais podem ser influenciados por atividades antrópicas. As mudanças nas condições geomorfológicas podem desencadear um aumento da descarga de sedimentos, mudanças na geometria do canal e nas áreas de inundações inativas, bem como perda da diversidade de habitats (Sullivan et al., 2006).

O Cerrado é alvo de intensa modificação causada pela fragmentação, representado por ilhas inseridas em uma matriz de agroecossistemas. Nesse contexto de exploração, os ambientes aquáticos têm sofrido fortes pressões, principalmente devido aos usos múltiplos da água. O Cerrado é o segundo maior bioma brasileiro, ocupando originalmente quase 2 milhões de km² (Ratter et al., 1997), cuja composição é formada por savanas, matas, campos e matas de galeria (Klink \& Machado 2005). Seu clima é caracterizado por estações de chuva e seca bem definidas, com temperaturas que variam entre 18 e $28^{\circ} \mathrm{C}$ e precipitação entre 800 e $2000 \mathrm{~mm}$ anuais (Ratter et al., 1997). Seu solo é, predominantemente, antigo, intemperizado e ácido (Klink \& Machado, 2005), o que facilita processos erosivos. A importância ecológica do Cerrado reside, principalmente, no fato de que os principais tributários das bacias do Paraguai e do São Francisco nascem nele, assim como alguns tributários da bacia Amazônica (Jepson, 2005).

Diante do exposto, o objetivo deste trabalho foi avaliar efeitos do uso do solo sobre atributos de riachos de cabeceira na bacia do Rio Cuiabá, Mato Grosso, uma vez que, nos últimos 30 anos, o Cerrado tem sofrido severos impactos antrópicos. 


\section{Materiais E Métodos}

\section{Área de Estudo}

A bacia do Rio Cuiabá está localizada entre os paralelos $14^{\circ} 18^{\prime}$ e $17^{\circ} 00^{\prime}$ e os meridianos $54^{\circ} 40^{\prime}$ e $56^{\circ} 55^{\prime}$, totalizando aproximadamente 29.000 $\mathrm{km}^{2}$ de área (Mato Grosso, 1997). Sua área é constituída pela planície do Pantanal e pelo planalto circunvizinho (Libos et al., 2005). O Rio Cuiabá é o maior afluente do Alto Rio Paraguai, que é a espinha dorsal do Pantanal. A maior parte da ameaça ao Pantanal advém de atividades agropecuárias desenvolvidas em seu entorno, no planalto, onde se localizam as suas nascentes, cuja vegetação pertence ao bioma Cerrado.

Os riachos amostrados são afluentes da Bacia do Rio Cuiabá e estão localizados entre os Paralelos $15^{\circ}$ e $16^{\circ}$ de latitude sul e Meridianos $55^{\circ} \mathrm{e}$ $56^{\circ}$ a oeste de Greenwich (Figura 1 e Tabela 1).

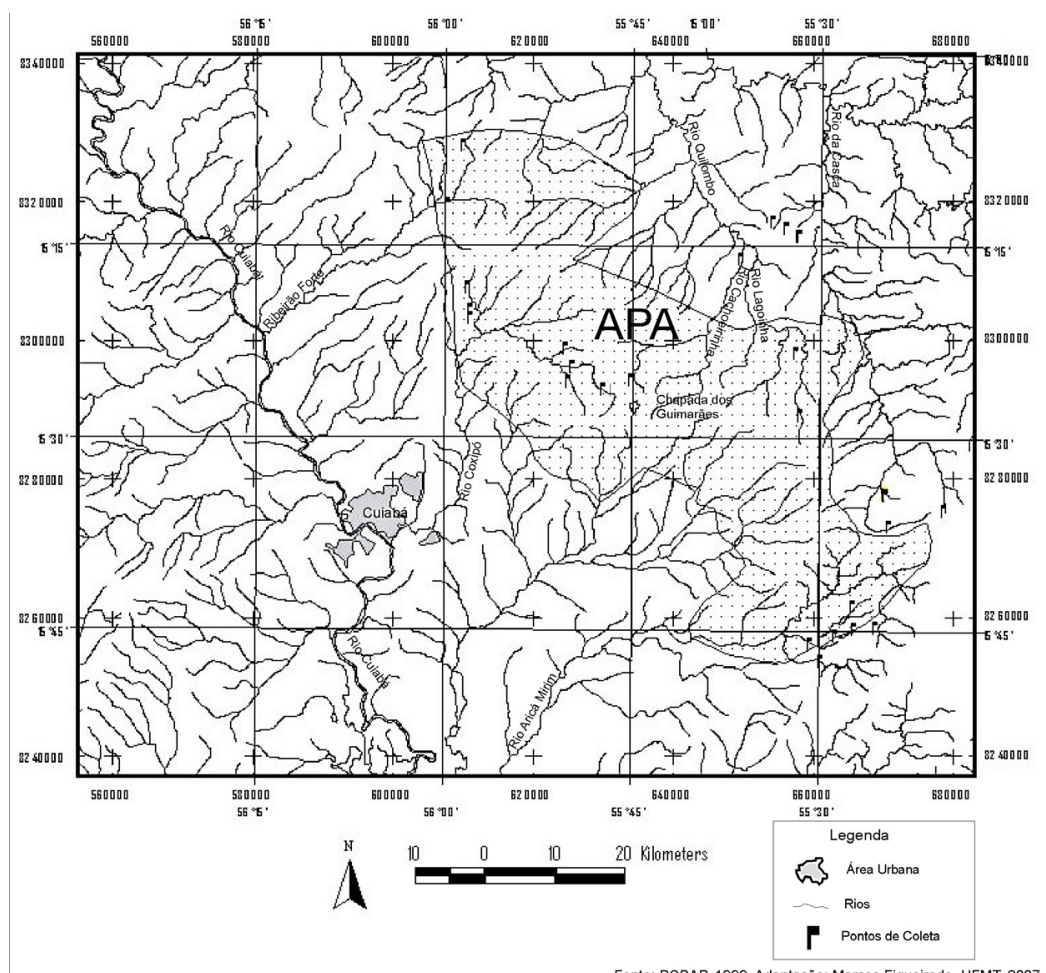

Fonte: PCBAP, 1999. Adaptação: Marcos Figueiredo, UFMT, 2007

Figura 1. Localização dos riachos amostrados na Bacia do Rio Cuiabá, Mato Grosso. 
Tabela 1. Coordenadas Geográficas dos riachos amostrados na Bacia do Rio Cuiabá, Mato Grosso.

\begin{tabular}{|c|c|c|}
\hline Pontos & $\mathrm{X}(\mathrm{W})$ & $\mathrm{Y}(\mathrm{S})$ \\
\hline 1 & $55^{\circ} 24^{\prime} 45.092^{\prime \prime}$ & $15^{\circ} 34^{\prime} 21.825^{\prime \prime}$ \\
\hline 2 & $55^{\circ} 20^{\prime} 00.725^{\prime \prime}$ & $15^{\circ} 35^{\prime} 45 \cdot 322^{\prime \prime}$ \\
\hline 3 & $55^{\circ} 24^{\prime} 21.944^{\prime \prime}$ & $15^{\circ} 36^{\prime} 56.820^{\prime \prime}$ \\
\hline 4 & $55^{\circ} 31^{\prime} 31.065^{\prime \prime}$ & $15^{\circ} 28^{\prime} 20.326^{\prime \prime}$ \\
\hline 5 & $55^{\circ} 31^{\prime} 51.690^{\prime \prime}$ & $15^{\circ} 23^{\prime} 25.931^{\prime \prime}$ \\
\hline 6 & $55^{\circ} 47^{\prime} 17.235^{\prime \prime}$ & $15^{\circ} 26^{\prime} 22.184^{\prime \prime}$ \\
\hline 7 & $55^{\circ} 45^{\prime} 01.622 "$ & $15^{\circ} 25^{\prime} 38.836^{\prime \prime}$ \\
\hline 8 & $55^{\circ} 58^{\prime} 30.931^{\prime \prime}$ & $15^{\circ} 07^{\prime} 20.132 "$ \\
\hline 9 & $55^{\circ} 58^{\prime} 07.463^{\prime \prime}$ & $15^{\circ} 18^{\prime} 25.272 "$ \\
\hline 10 & $55^{\circ} 59^{\prime} 49.355^{\prime \prime}$ & $15^{\circ} 11 ' 50.562 "$ \\
\hline 11 & $55^{\circ} 27^{\prime} 16.102 "$ & $15^{\circ} 43^{\prime} 14.444^{\prime \prime}$ \\
\hline 12 & $55^{\circ} 30^{\prime} 40.180^{\prime \prime}$ & $15^{\circ} 46^{\prime} \circ 8.047^{\prime \prime}$ \\
\hline 13 & $55^{\circ} 25^{\prime} 27.192^{\prime \prime}$ & $15^{\circ} 44^{\prime} 56.022 "$ \\
\hline 14 & $55^{\circ} 27^{\prime} \circ 6.086^{\prime \prime}$ & $15^{\circ} 45^{\prime} 01.807^{\prime \prime}$ \\
\hline 15 & $55^{\circ} 28^{\prime} 35.490^{\prime \prime}$ & $15^{\circ} 45^{\prime} 32.050^{\prime \prime}$ \\
\hline 16 & $55^{\circ} 29^{\prime} 45.782 "$ & $15^{\circ} 47^{\prime} 30.185^{\prime \prime}$ \\
\hline 17 & $55^{\circ} 57^{\prime} 55.811 "$ & $15^{\circ} 20^{\prime} 45.152^{\prime \prime}$ \\
\hline 18 & $55^{\circ} 57^{\prime} 58.15^{\prime \prime}$ & $15^{\circ} 20^{\prime} 12.262 "$ \\
\hline 20 & $55^{\circ} 50^{\prime} 19.995^{\prime \prime}$ & $15^{\circ} 23^{\prime} 05.701 "$ \\
\hline 21 & $55^{\circ} 49^{\prime} 47.088^{\prime \prime}$ & $15^{\circ} 24^{\prime} 36.351 "$ \\
\hline 22 & $55^{\circ} 50^{\prime} 02.374^{\prime \prime}$ & $15^{\circ} 25^{\prime} 44 \cdot 378^{\prime \prime}$ \\
\hline 23 & $55^{\circ} 31^{\prime} 38.899 "$ & $15^{\circ} 14^{\prime} 21.484^{\prime \prime}$ \\
\hline 24 & $55^{\circ} 31^{\prime} 44.436^{\prime \prime}$ & $15^{\circ} 14^{\prime} 15.696 "$ \\
\hline 25 & $55^{\circ} 32^{\prime} 41.270^{\prime \prime}$ & $15^{\circ} 13^{\prime} 4^{2} .324^{\prime \prime}$ \\
\hline 26 & $55^{\circ} 33^{\prime} 45.250^{\prime \prime}$ & $15^{\circ} 13^{\prime} 11.987^{\prime \prime}$ \\
\hline 27 & $55^{\circ} 36^{\prime} 19.309 "$ & $15^{\circ} 16^{\prime} 03.919^{\prime \prime}$ \\
\hline
\end{tabular}




\section{Coleta de dados}

Ao total, 26 riachos de $1^{\mathrm{a}}$ e $2^{\mathrm{a}}$ ordens foram amostrados em junho e dezembro de 2005. A partir da microbacia dos riachos amostrados, o uso do solo foi caracterizado como solo exposto, pastagem, monocultura e vegetação nativa por imagem de satélite, assim como a largura da mata ciliar foi medida. Foram utilizadas as bandas RGB (345) do satélite LANDSAT 5 TM de 05/08/2006 (21 L) e o programa ENVI 4.3. Para tanto, foi realizado realce linear nas bandas, composição das bandas em uma única imagem, recorte das microbacias, classificação supervisionada automática por máxima verossimilhança, quantificação da porcentagem das categorias de uso do solo e medição da largura da mata ciliar.

A largura do canal do riacho foi calculada como a média de 06 (seis) medidas transversais, uma a cada 10 metros. A profundidade foi medida com uma trena graduada, a cada $20 \mathrm{~cm}$, ao longo dos $\circ 6$ (seis) pontos utilizados para as tomadas de medidas de largura. Os tipos de substratos amostrados foram categorizados em: raiz (R), tronco (T), cascalho $(C)$, areia $(A)$, matação $(M)$, argila $(G)$, folha $(F)$, silte $(S)$, lage (L). Foi caracterizada como alteração hidrológica a presença de represamento artificial a montante ou jusante, cachoeira e/ou sumidouro.

Em três pontos de cada riacho, as velocidades máxima, mínima e média foram medidas com um fluxímetro (MiniAir 2 Schiltknecht), bem como o oxigênio dissolvido em porcentagem (\%) com um oximêtro (YSI EcoSense DO200); o pH com o peagâmetro (Oakton 300) e a condutividade e a temperatura em NTU e ${ }^{\circ} \mathrm{C}$ com o condutivímetro (Orion $115 \mathrm{~A}+$ ) em cada riacho.

\section{Análise de dados}

O programa MINITAB 15 foi utilizado para realizar as análises estatísticas. Primeiramente, foi feito um teste de normalidade para todas as variáveis. Nas variáveis que apresentavam zero foi somado 0,5 e, em seguida, foi realizada uma transformação logarítmica. Os dados foram padronizados pela subtração da média e divisão pelo desvio padrão de todas as variáveis, uma vez que a escala entre elas era distinta. Correlação, regressão linear simples e múltipla foram usadas para construir modelos exploratórios, ligando usos do solo a condições morfológicas dos riachos. 


\section{Resultados}

Houve correlação positiva (p-valor < 0,05) entre: oxigênio dissolvido e solo exposto (46,1\%); oxigênio dissolvido e folha (39,6\%); $\mathrm{pH}$ e pastagem $(44,2 \%)$; velocidade e largura do canal $(52,6 \%)$; largura do canal e agricultura (40,3\%); largura do canal e cascalho (47,3\%); mata ciliar e folha $(48,2 \%)$; vegetação nativa e silte $(47,8 \%)$; agricultura e cascalho $(37,8)$; raiz e areia (39,2\%); tronco e areia (37,7\%); matacão e silte $(41,3 \%)$.

Correlações negativas significativas ( $\mathrm{p}$-valor $<0,05$ ) foram encontradas entre: condutividade e profundidade do canal $(-53,4 \%)$; condutividade e solo exposto $(-38,9 \%)$; condutividade e cascalho $(-44,5 \%)$; turbidez e oxigênio dissolvido (-45\%); oxigênio dissolvido e matacão $(-52,4 \%)$; temperatura e largura do canal (-45,5\%); $\mathrm{pH}$ e profundidade (-40,6\%); $\mathrm{pH}$ e agricultura (-40,4\%); pH e alteração hidrológica $(-45,4)$; pH e argila $(-37,6 \%)$; velocidade e vegetação nativa $(-38,6 \%)$; velocidade e silte $(-53,9 \%)$; profundidade do canal e folha (-41,1\%); profundidade do canal e silte $(-38 \%)$; largura do canal e raiz $(-51,2 \%)$; mata ciliar e argila $(-49,5 \%)$; vegetação nativa e agricultura $(-49,4)$; vegetação nativa e solo exposto $(-74,1 \%)$; agricultura e pastagem $(-40,7 \%)$; agricultura e silte $(-41,1)$; solo exposto e matacão $(-38,2)$; pastagem e alteração hidrológica $(-37,5)$; areia e argila $(-40,4)$.

A turbidez afetou negativamente o oxigênio dissolvido em riachos de cabeceira (Figura 2), assim como a largura do canal afetou a temperatura (Figura 3) e a largura da mata ciliar afetou positivamente a quantidade de folhas (Figura 4).

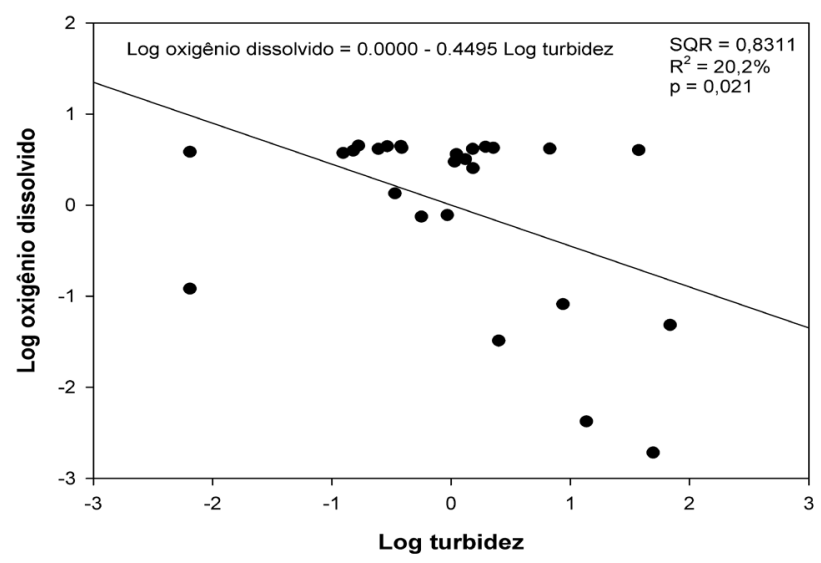

Figura 2. Efeito da turbidez sobre o oxigênio dissolvido em riachos de cabeceira na bacia do Rio Cuiabá, Mato Grosso, 2005. 


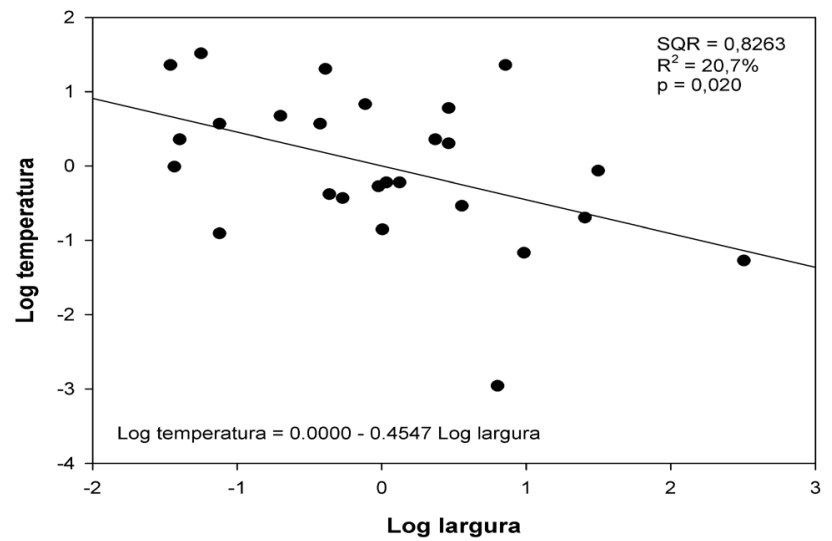

Figura 3. Efeito da largura sobre a temperatura em riachos de cabeceira na bacia do Rio Cuiabá, Mato Grosso, 2005.

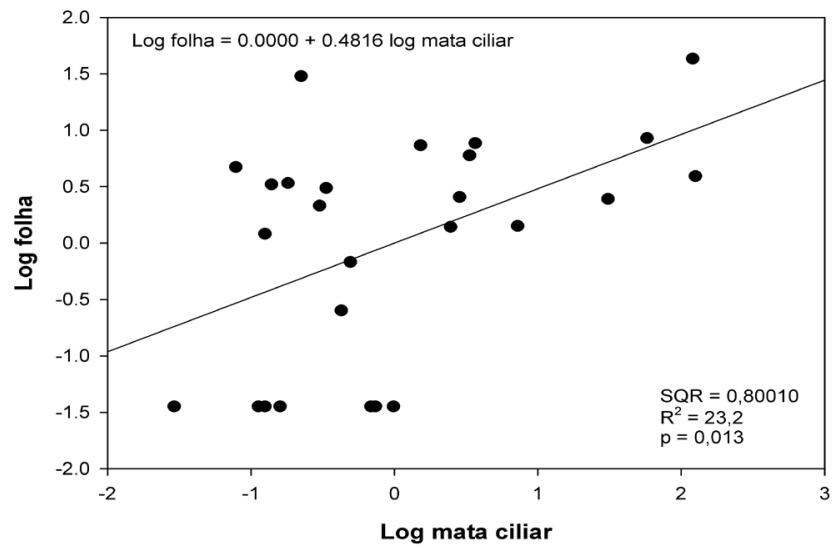

Figura 4. Efeito da mata ciliar sobre o substrato folha em riachos de cabeceira na bacia do Rio Cuiabá, Mato Grosso, 2005.

As variáveis que mais afetaram o $\mathrm{pH}$ foram a profundidade $(16,51 \%)$ e os substratos silte $(12,03 \%)$ e argila (10,07\%) (Tabela 1). O modelo foi considerado adequado após análise residual, sendo que o valor do $\beta_{\circ}=$ 0,325 e o coeficiente de determinação apresentou valor de $64 \%\left(R^{2}=64\right)$ com um desvio padrão amostral de $0,7748(\mathrm{~s}=0,7748), \mathrm{F}_{10,15}=2,66 \mathrm{e}$ $\mathrm{p}$-valor de $0,042(\mathrm{p}=0,042)$.

Tabela 1. Análise de regressão linear múltipla entre pH e profundidade, silte, argila, pastagem, agricultura, alteração hidrológica, mata ciliar, 
areia, tronco e folha dos riachos de cabeceira da bacia do Rio Cuiabá, Mato Grosso, 2005.

\begin{tabular}{cccc}
\hline Variável & Coeficiente & Desvio padrão & $\mathbf{R}^{2}$ \\
\hline Profundidade & $-0,0244$ & 0,2136 & 16,51 \\
Silte & 0,5600 & 0,2502 & 12,03 \\
Argila & $-0,2231$ & 0,2129 & 10,07 \\
Pastagem & 0,4767 & 0,2498 & 8,69 \\
Agricultura & 0,1587 & 0,2243 & 8,00 \\
Alteração hidrológica & $-0,5632$ & 0,4608 & 5,31 \\
Mata ciliar & 0,2285 & 0,2299 & 2,14 \\
Areia & 0,0363 & 0,2270 & 0,58 \\
Tronco & 0,0650 & 0,1917 & 0,48 \\
Folha & $-0,1251$ & 0,2012 & 0,15 \\
\hline
\end{tabular}

As variáveis que mais afetaram o oxigênio dissolvido foram o substrato matacão $(27,47 \%)$ e o solo exposto $(21,29 \%)$ (Tabela 2$)$. O modelo foi considerado adequado após análise residual, sendo que o valor do $\beta$ 。 $=0,000$ e o coeficiente de determinação apresentou valor de $57,8 \%\left(\mathrm{R}^{2}=\right.$ $57,8)$, com um desvio padrão amostral de $0,745^{2}\left(\mathrm{~s}=0,745^{2}\right), \mathrm{F}_{6,19}=4,34$ e p-valor de $0,006(\mathrm{p}=0,006)$.

Tabela 2. Análise de regressão linear múltipla entre oxigênio dissolvido e os substratos matacão e folha, condutividade, vegetação nativa, solo e turbidez dos riachos de cabeceira da bacia do Rio Cuiabá, Mato Grosso.

\begin{tabular}{cccc}
\hline Variável & Coeficiente & Desvio padrão & $\mathbf{R}^{2}$ \\
\hline Matacão & $-0,3117$ & 0,1838 & 27,47 \\
Solo exposto & 0,6790 & 0,2473 & 21,29 \\
Folha & 0,1951 & 0,1725 & 5,09 \\
Turbidez & $-0,2032$ & 0,1630 & 3,45 \\
Vegetação nativa & 0,3718 & 0,2323 & 0,29 \\
Condutividade & 0,2074 & 0,1698 & 0,19 \\
\hline
\end{tabular}

As variáveis que mais afetaram a condutividade foram a profundidade $(28,55 \%)$ e o solo exposto $(11,81 \%)$ (Tabela 3$)$. O modelo foi considerado adequado após análise residual, sendo que o valor do $\beta_{\circ}=0,0 \circ 0$ e o coe- 
ficiente de determinação apresentou valor de 50,7\% $\left(\mathrm{R}^{2}=50,7\right)$ com um desvio padrão amostral de $0,7662(\mathrm{~s}=0,7662), \mathrm{F}_{4,21}=5,40$ e p-valor de $0,004(p=0,004)$.

Tabela 3. Análise de regressão linear múltipla entre condutividade e profundidade, solo exposto, o substrato cascalho e vegetação nativa dos riachos de cabeceira da bacia do Rio Cuiabá, Mato Grosso.

\begin{tabular}{cccc}
\hline Variável & Coeficiente & Desvio padrão & $\mathbf{R}^{2}$ \\
\hline Profundidade & $-0,4642$ & 0,1614 & 28,55 \\
Solo exposto & $-0,5358$ & 0,2291 & 11,81 \\
Cascalho & $-0,2726$ & 0,1657 & 5,23 \\
Vegetação nativa & $-0,3423$ & 0,2322 & 5,10 \\
\hline
\end{tabular}

A largura e a vegetação nativa afetaram $27,62 \%$ e $7,44 \%$ a velocidade, respectivamente (Tabela 4). O modelo foi considerado adequado após análise residual, sendo que o valor do $\beta_{\circ}=0,000$ e o coeficiente de determinação apresentou valor de 35,1\% $\left(\mathrm{R}^{2}=35,1\right)$, com um desvio padrão amostral de 0,8401 ( $\mathrm{s}=0,8401), \mathrm{F}_{2,23}=6,21$ e p-valor de 0,007 $(\mathrm{p}=0,007)$.

Tabela 4. Análise de regressão linear múltipla entre velocidade e largura e vegetação nativa dos riachos de cabeceira da bacia do Rio Cuiabá, Mato Grosso.

\begin{tabular}{cccc}
\hline Variável & Coeficiente & Desvio padrão & $\mathbf{R}^{2}$ \\
\hline Largura & 0,4613 & 0,1726 & 27,62 \\
Vegetação nativa & $-0,2803$ & 0,1726 & 7,44 \\
\hline
\end{tabular}

O substrato raiz, a agricultura e o substrato cascalho afetaram 31,11\%, $16,24 \%$ e 3,05\% a largura, respectivamente (Tabela 5). O modelo foi considerado adequado após análise residual, sendo que o valor do $\beta_{\circ}=0,000 \mathrm{e}$ o coeficiente de determinação apresentou valor de 50,4\% $\left(\mathrm{R}^{2}=50,4\right) \mathrm{com}$ um desvio padrão amostral de $0,7507(\mathrm{~s}=0,7507), \mathrm{F}_{3,22}=7,45$ e p-valor de $0,001(p=0,001)$. 
Tabela 5. Análise de regressão linear múltipla entre largura e o substrato raiz, agricultura e o substrato cascalho dos riachos de cabeceira da bacia do Rio Cuiabá, Mato Grosso.

\begin{tabular}{cccc}
\hline Variável & Coeficiente & Desvio padrão & $\mathbf{R}^{2}$ \\
\hline Raiz & $-0,5006$ & 0,1596 & 31,11 \\
Agricultura & 0,3807 & 0,1665 & 16,24 \\
Cascalho & 0,1996 & 0,1714 & 3,05 \\
\hline
\end{tabular}

As variáveis que mais afetaram o substrato silte foram a velocidade $(29,10 \%)$, solo exposto $(17,88 \%)$ e largura $(11,16 \%)$ (Tabela 6). O modelo foi considerado adequado após análise residual, sendo que o valor do $\beta$ 。 $=0,000$ e o coeficiente de determinação apresentou valor de 79,8\% $\left(\mathrm{R}^{2}\right.$ $=79,8)$, com um desvio padrão amostral de $0,5295(\mathrm{~s}=0,5295), \mathrm{F}_{7,18}=$ 10,16 e p-valor de $0,000(\mathrm{p}=0,000)$.

Tabela 6. Análise de regressão linear múltipla entre o substrato silte e velocidade, solo exposto, largura, profundidade, vegetação nativa, agricultura e o substrato matacão dos riachos de cabeceira da bacia do Rio Cuiabá, Mato Grosso.

\begin{tabular}{cccc}
\hline Variável & Coeficiente & Desvio padrão & $\mathbf{R}^{2}$ \\
\hline Velocidade & $-0,4449$ & 0,1413 & 29,10 \\
Solo exposto & 0,6666 & 0,1670 & 17,88 \\
Largura & 0,3103 & 0,1367 & 11,16 \\
Profundidade & $-0,2341$ & 0,1130 & 9,82 \\
Vegetação nativa & 0,6189 & 0,1767 & 6,80 \\
Agricultura & $-0,2386$ & 0,1359 & 3,06 \\
Matacão & 0,296 & 0,1244 & 1,98 \\
\hline
\end{tabular}

Os substratos raiz, argila e tronco afetaram $15,34 \%, 8,42 \%$ e $6,52 \%$ o substrato areia, respectivamente (Tabela 7). O modelo foi considerado adequado após análise residual, sendo que o valor do $\beta_{\circ}=0,000$ e o coeficiente de determinação apresentou valor de $30,3 \%\left(\mathrm{R}^{2}=30,3\right)$, com um desvio padrão amostral de $0,8898(\mathrm{~s}=0,8898), \mathrm{F}_{3,22}=3,19$ e $\mathrm{p}$-valor de $0,044(p=0,044)$. 
Tabela 7. Análise de regressão linear múltipla entre o substrato areia e os substratos raiz, argila e tronco dos riachos de cabeceira da bacia do Rio Cuiabá, Mato Grosso.

\begin{tabular}{cccc}
\hline Variável & Coeficiente & Desvio padrão & $\mathbf{R}^{2}$ \\
\hline Raiz & 0,2791 & 0,1856 & 15,34 \\
Argila & 0,2218 & 0,1913 & 8,42 \\
Tronco & $-0,2733$ & 0,1898 & 6,52 \\
\hline
\end{tabular}

A largura e a agricultura afetaram $22,36 \%$ e $4,19 \%$ o substrato cascalho, respectivamente (Tabela 8). O modelo foi considerado adequado após análise residual, sendo que o valor do $\beta_{\circ}=0,000$ e o coeficiente de determinação apresentou valor de $26,5 \%\left(R^{2}=26,5\right)$, com um desvio padrão amostral de $0,8935(\mathrm{~s}=0,8935), \mathrm{F}_{2,23}=4,16$ e p-valor de $0,029(\mathrm{p}=0,029)$.

Tabela 8. Análise de regressão linear múltipla entre o substrato cascalho e largura e agricultura dos riachos de cabeceira da bacia do Rio Cuiabá, Mato Grosso.

\begin{tabular}{cccc}
\hline Variável & Coeficiente & Desvio padrão & $\mathbf{R}^{2}$ \\
\hline Largura & 0,3827 & 0,1953 & 22,36 \\
Agricultura & 0,2236 & 0,1953 & 4,19 \\
\hline
\end{tabular}

\section{Discussão}

Os atributos físicos do canal e os químicos da água dos riachos de cabeceira da bacia do Rio Cuiabá foram afetados direta e indiretamente pelas atividades agropecuárias. É importante destacar que não somente cobertura do solo em áreas de vegetação, mas também a cobertura do solo na área de drenagem é fator importante que afeta as condições dos riachos (Heartsill-Scalley \& Aide, 2003).

Mudanças na cobertura do solo de floresta para agricultura e pastagem frequentemente alteram a mata ciliar, que modifica as condições físicas dos riachos (Heartsill-Scalley \& Aide, 2003). Os riachos são sistemas abertos que exercem influências e são influenciados pelo ambiente terrestre adjacente. Portanto, riachos de cabeceira são os ecossistemas mais vulneráveis aos impactos antropogênicos (Menéndez et al., 2012). Vale ressaltar que, nenhum outro fator está tão relacionado às variações 
nas condições ambientais de sistemas lóticos quanto a vegetação ripária ou ciliar (Krupek et al., 2010).

A mata ciliar é o principal fator que influencia a entrada de serapilheira nos riachos, seja diretamente ou transportada lateralmente a partir do chão da floresta (Naiman \& Decamps, 1997). A mata ciliar produz grande quantidade de liteira na forma de folhas (Nunes \& Pinto, 2007). Como os riachos de cabeceira dependem de energia alóctone (Cummins, 1974), as folhas podem fornecer parte da energia externa que dão entrada nesses riachos. As atividades agrícolas exercem pressão na redução da largura da mata ciliar (Donato et al., 2010), provocando a diminuição da entrada de energia em riachos de cabeceira e, consequentemente, a perda de biodiversidade de macroinvertebrados aquáticos e peixes.

A mata ciliar contribui para a alta biodiversidade, protege o canal dos riachos de mudanças temporais, regula a temperatura da água dos riachos, filtra e retem nutrientes e fornece habitat, refúgio e comida para organismos (Naiman et al., 1993; Naiman \& Décamps, 1997). Além disso, ela tem influência direta na qualidade e quantidade de luz que chega até os corpos d'água, levando por sua vez a mudanças na temperatura da água (Krupek et al., 2010). A mata ciliar é essencia para proteger a qualidade das águas dos rios e, pelo efeito de sombreamento, intercepta e absorve a radiação solar, contribuindo para a estabilidade térmica, evitando excessivos aquecimentos diurnos e resfriamentos noturnos (Brown \& Krygier, 1970).

O aumento do canal do riacho provoca exposição à radiação solar e, consequentemente, aumento da temperatura da água (Fritzsons et al., 2005; Nakamura \& Dokai, 1989). Radiação solar direta na superfície da água é uma fonte de energia térmica para os riachos (Sinokrot \& Stefan, 1993), mas outras fontes e fluxos de energia também contribuem para a temperatura do riacho em um dado ponto, tais como evaporação, advecção e condução (Johnson \& Jones, 2000). Em estudos do potencial dos efeitos das mudanças climáticas globais em ecossistemas de água doce, a temperatura da água é um fator primário (Mohseni \& Stefan, 1999), que é influenciado por mudanças no uso do solo dos riachos (Johnson $\&$ Jones, 2000).

O aumento da profundidade do canal do riacho está associado ao aumento da quantidade de água e de seu poder de diluição, o que afeta a concentração de íons carbonato e bicarbonato do Rio Cuiabá, proveniente das 
rochas calcárias da região das nascentes, com diminuição da alcalinidade (Figueiredo, 1996) e, consequentemente de seu pH. O aumento da quantidade de água no canal do riacho ainda afeta o transporte de partículas e íons dissolvidos, diminuindo sua condutividade. A baixa condutividade indica pouca influência antrópica na região (Smith \& Petrere, 2000).

O aumento da turbidez contribuiu para a diminuição do oxigênio dissolvido, uma vez que a presença de partículas em suspensão causa turbidez, limita a penetração de raios solares e restringe a atividade fotossintética que, por sua vez, reduz a reposição do oxigênio consumido por organismos em sistemas aquáticos (Lima, 2001).

O desmatamento das áreas adjacentes e a declividade do relevo em riachos de cabeceira aumentam a velocidade da água via escoamento superficial, provocando erosão (Tucci \& Clarke, 1997) e aumento na largura do canal dos riachos. Vale ressaltar que as principais causas de erosão são o desmatamento, as construções de estradas e o garimpo (Wantzen \& Pinto-Silva, 2006).

O movimento do sedimento em riachos é influenciado por uma série de fatores, incluindo fonte variável de sedimento, fluxos transitórios, tamanho variável de partículas e não uniformidade da geometria do canal e fluxo (Silva \& Santos, 2008; Poole, 2010). A vegetação das margens influencia significativamente na morfologia do canal dos riachos. Então, a largura do canal dos riachos é relacionada ao tipo de uso do solo e a largura aumenta, em resposta ao aumento da descarga (Zimmerman et al., 1967). Estudos de associação entre índices de integridade bióticas e qualidade ambiental indicam que a degradação, perda de habitat, fragmentação e poluição são as principais causas de perda de integridade biótica e abiótica em riachos (Machado et al., 2011).

Embora haja efeito do uso do solo sobre propriedades estruturais e funcionais de riachos, a gama de possíveis respostas do canal é muito variada. A velocidade e direção de mudanças na profundidade do canal, largura ou composição e o tamanho de sedimentos podem variar de acordo com alterações na descarga de água e sedimentos. Além da complexidade inerente dos riachos, as mudanças de uso da terra são normalmente distribuídas no tempo e no espaço, o que pode dificultar padrões de respostas explícitos através de testes estatísticos. Outros fatores que podem ter dificultado resultados estatísticos mais robustos são: (i) na estação seca, riachos de cerrado apresentam o nível de água estável, enquanto que na 
estação chuvosa ocorrem enchentes (Wantzen, 2003), tornando esses ambientes mais instáveis; (ii) inexistência de dados em escala temporal; (iii) inexistência de dados de escoamento superficial e descarga de água.

Agradecimentos

Esta pesquisa foi financiada pela FAPEMAT (4.2.2.83/02-2004-E), Centro de Pesquisa do Pantanal (CPP), e Ministério da Ciência e Tecnologia (MCT). N.G.M. agradece uma bolsa do CNPq.

\section{Bibliografia}

ALLAN, J.D. 1995. Stream Ecology: Structure and Function of Running Waters. Dordrecht. Netherland: Kluwer. p. 388.

ALLAN, J.D. 2004. Landscapes and Riverscapes: the influence of land use on stream ecosystems. Annual Review of Ecology Evolution and Systematics $35: 257-284$.

ANDERSON, N.H. \& SEDELL, J.R. 1979. Detritus processing by macroinvertebrates in stream ecosystems. Annual Review of Entomology 24:351-377.

CHURCH, M. 2002. Geomorphic thresholds in riverine landscape. Freshwater Biology 47: 541-557.

CUMMINS, K.W. 1974. Structure and function of stream ecosystems. Biosciences 24: 631-641.

DONATO, C.T.; LAMEIRA, W.J;; ALMEIDA, A.S. \& VIEIRA, I.C.G. 2010. Análise espacial dos fragmentos de mata ciliar no município de Peixe-boi. Pará. In: III Simpósio Brasileiro de Ciências Geodésicas e Tecnologias da Geoinformação. Recife - PE. 27-30 de Julho de 2010.

FAUSCH, K.D.; TORGERSEN, C.E.; BAXTER, C.V. \& LI, H.W. 2002. Landscapes to Riverscapes: Bridging tile Gap between Research and Conservation of Stream Fishes. BioScience 52: 483-498.

FIGUEIREDO, D.M.A. Influência dos fatores climáticos e geológicos e da ação antrópica sobre as principais variáveis físicas e químicas do Rio Cuiabá. 1996. (Dissertação de Mestrado). Instituto de Biociências. UFMT. 
HEARTSILL-SCALLEY, T. \& AIDE, T.M. 2003. Riparian vegetation and stream condition in a tropical agriculture-secondary forest mosaic. Ecological Applications 13(1): 225-234.

JEPSON, W. 2005. A disappearing biome? Reconsidering land-cover change in the Brazilian savanna. The Geographical Journal 171: 99-111.

JOHNSON, S.L. \& JONES, J.A. 2000. Stream temperature responses to forest harvest and debris flows in western Cascades, Oregon. Canadian Journal of Fishery Aquatic Science 57(2): 30-39.

JOHNSON, W.C. 2002. Riparian vegetation diversity along regulated rivers: contribution of novel and relict habitats. Freshwater Biology 47: 749-759.

KARR, J.R. \& SCHLOSSER, I.J. 1978. Water resource and the land-water interface. Science 201: 229-234.

KRUPEK, R.A.; BRANCO, C.C.Z. \& PERES, C.K. 2010. Variação sazonal das variáveis físicas e químicas em riachos de duas bacias da região centro-sul do estado do Paraná, sul do Brasil. Ambiência 6: 297-310.

LIMA, E.B.N.R. Modelagem Integrada para Gestão da Qualidade da água na bacia do Rio Cuiabá. 2001. Tese (Doutorado no Programa de Pós-Graduação de Engenharia). Universidade Federal do Rio de Janeiro. Rio de Janeiro.

LOWE, W.H.; LIKENS, G.E. \& POWER, M.E. 2006. Linking Scales in Stream Ecology. BioScience 56(7): 591-597.

MACHADO, N.G.; VENTICINQUE, F.M. \& PENHA, J.M.F. 2011. Effect of environmental quality and mesohabitat structure on a Biotic Integrity Index based on fish assemblages of cerrado streams from Rio Cuiabá basin, Brazil. Brazilian Journal of Biology 71 (3): 577-586.

MENÉNDEZ, M.; DESCALS, E.; RIERA, T. \& MOYA, O. 2012. Effect of small reservoirs on leaf litter decomposition in Mediterranean headwater streams. Hydrobiologia 691: 135-146. 
MOHSENI, O. \& STEFAN, H.G. 1999. Stream temperature/air temperature relationship: a physical interpretation. Journal of Hydrology 218 (3-4): 128-141.

NAIMAN, R.J.; DÉCAMPS, H. 1997. The ecology of the interfaces; riparian zones. Annual Review of Ecology and Systematics 28: 621-658.

NAKAMURA, F. \& DOKAI, T. 1989. Estimation of the effect of riparian Forest on stream temperature based on heat budget. Journal of Japanese Forestry Society 71: 387-394.

NUNES, F.P. \& PINTO, M.T.C. 2007. Produção de serapilheira em mata ciliar nativa e reflorestada no alto São Francisco. Minas Gerais. Biota Neotropical 7 (3): 97-102.

OLIVEIRA, R.S.; BEZERRA, L.; DAVIDSON, E.A.; PINTO, F.; KLINK, C.A.; NEPSTAD, D.C. \& MOREIRA, A. 2005. Deep root function in soil water dynamics in cerrado savannas of central Brazil. Functional Ecology 19: $574-581$.

PINTO, A.L.; OLIVEIRA, G.H. \& PEREIRA, G.A. 2010. Avaliação da eficiência da utilização do oxigênio dissolvido como principal indicador da qualidade das águas superficiais da bacia do córrego Bom Jardim. Brasilândia/MS. Revista de Geografia, Meio Ambiente e Ensino o1 (01): 69-82.

POFF, N.L. 1997. Landscape filters and species traits: towards mechanistic understanding and prediction in stream ecology. Journal of the North American Benthological Society 16(2): 391-409.

POOLE, G.C. 2010. Stream hydrogeomorphology as a physical science basis for advances in stream ecology. Journal of the North American Benthological Society 29(1): 12-25.

PRINGLE, C. 2003. What is hydrologic connectivity and why is it ecologically important? Hydrological Processes 17: 2685-2689.

SILVA, R.M. \& SANTOS, C.A.G. 2008. Estimativa da produção de sedimentos mediante uso de um modelo hidrossedimentológico acoplado ao SIG. Revista Brasileira de Engenharia Agrícola e Ambiental 12 (5): 520526. 
SINOKROT, B.A. \& STEFAN, H.G. 1993. Stream temperature dynamics: Measurements and modeling. Water Resources Research 29 (7): 22992312.

SMITH, W.S. \& PETRERE Jr., M. 2000. Caracterização limnológica da bacia de drenagem do rio Sorocaba. São Paulo. Brasil. Acta Limnologica Brasiliensis 12: 15-27.

SULLIVAN, S.M.P.; WATZIN, M.C. \& HESSION, W.C. 2006. Influence of stream geomorphic condition on fish communities in Vermont, U.S.A. Freshwater Biology 51: 1811-1826.

TUCCI, C.E.M. \& CLARKE, R.T. 1997. Impacto das mudanças da cobertura vegetal no escoamento: revisão. Revista Brasileira de Recursos Hídri$\cos 2(1): 135^{-152 .}$

WANTZEN, K.M. \& PINTO-SILVA, V. 2006. Uso de Substratos Artificiais para Avaliação do Impacto do Assoreamento sobre Macroinvertebrados Bentônicos em um Córrego de Cabeceira no Pantanal do Mato Grosso, Brasil. Revista Brasileira de Recursos Hídricos 11 (1): 99-107.

WANTZEN, K.M. 2003. Cerrado streams - characteristics of a threatened freswater ecosystem type on the Tertiary Shields of Centra South America. Amazoniana XVII (3/4): 481-502.

WANTZEN, K.M.; SIQUEIRA, A.; NUNES DA CUNHA, C. \& SÁ, M.F. 2006. Conservation scheme for forest-stream-ecosystems of the Brazilian Cerrado and similar biomes in the seasonal tropics. Aquatic Conservation 16: 713-732.

WIENS, J.A. 2002. Riverine landscapes: taking landscape ecology into the water. Freshwater Biology 47: 501-515.

ZIMMERMAN, R.C.; GOODLETT, J.C. \& COMER, G.H. 1967. The influence of vegetation on channel form of small streams. International Association of Scientific Hydrology 75: 255-275.

Submetido em: 18/07/2011

Aceito em: 03/12/2012 